\title{
Design of Porous-Microchannel Heat Sinks with Different Porous Configurations
}

\author{
Tu-Chieh Hung, Yu-Xian Huang, and Wei-Mon Yan
}

\begin{abstract}
The models of the porous-microchannel heat sinks (porous-MCHSs) with different porous configurations are established in this work. Thermal performances of the porousMCHSs are investigated with the Reynolds number ranging from 45 to 1350 . The results indicate that, compared with the results of non-porous MCHS, the thermal performances can be enhanced by using the porous configurations and increase with an increase in the Reynolds number, except for the case with a lower Reynolds number. Both the sandwich and the trapezoidal distribution designs have the best heat transfer efficacy, cooling performance and convective performance. In particular, the thermal performance for rectangular, outlet enlargement, thin rectangular, or block distribution design is not necessarily better than that without porous medium under lower pumping power. Finally, the sandwich distribution design is the best design of the porous-MCHSs by considering the thermal performance along with the pressure drop.
\end{abstract}

Index Terms-Porous-microchannel, heat sink (porousMCHS).

\section{INTRODUCTION}

The 3-D numerical model to investigate the effects of the porous configuration designs on the thermal performance of the porous-microchannel heat sinks (porous-MCHSs, as shown in Fig. 1) were proposed in this paper. The porousmicrochannel heat sinks make use of the insertion of a porous metallic medium into the microchannel to raise both the surface contact area-to-volume ratio with the coolant and the local velocity mixing of the coolant. Higher contact surface area-to-volume ratio and velocity mixing of the fluid provide a better convective heat transfer effect [1], [2]. It is known [3]-[10] that the heat transfer performance of porousMCHSs can be improved if the configurations of porosity are properly designed. The heat transfer improvement makes the porous-MCHSs possible of implementing a task of micro-scale electronics cooling [1], [2], [10].

Many efforts have been devoted to improve the cooling performance of porous channels [1]-[10]. A sintered porous heat sink for the cooling of high-powered compact microprocessors was examined experimentally by Singh et al. [1]. The forced convection in porous rectangular ducts was performed by Calmidi and Mahajan [3] and Haji-Sheikh

Manuscript received April 14, 2015; revised July 20, 2015. This work was supported in part by the National Science Council, R. O. C.

Tu-Chieh Hung is with the Department of Mechanical Engineering, R.O.C Military Academy, Kaohsiung 83059, Taiwan (e-mail: christ.rich@msa.hinet.net).

Yu-Xian Huang is with Delta Electronics, Tainan County 74144 Taiwan (e-mail: frank-303@yahoo.com.tw).

Wei-Mon Yan is with the Department of Energy and Refrigerating AirConditioning Engineering, National Taipei Universi, Taiwan. et al. [4]. Zehforoosh and Hossainpour [5] investigated numerically the laminar forced convection in a partially porous channel using four dissimilar porous blocks attached to strip heat sources at the bottom wall. The fluid flow and heat transfer characteristics of a porous heat sink situated in a rectangular channel with bypass spaces was carried out experimentally by Jeng et al. [6]. Tamayol et al. [7] investigated the pressure drop in microfluidic minichannels filled with porous media formed by square arrays of microcylinders. Alfieri et al. [8] presented a fundamental hydrothermal investigation of the next generation interlayer integrated water cooled three-dimensional chip stacks, with high volumetric heat generation. Wan et al. [9] proposed a novel thermal management method of high-power lightemitting diodes (LEDs) by using a porous micro heat-sink system. The forced convection heat transfer in channels filled with sintered bronze media was examined by Jiang et al. [11], [12]. The heat transfer and pressure drop in a rectangular channel with sintered porous stainless steel inserts of different porosities for the purpose of cooling mini-devices was investigated by Hetsroni et al. [13]. Tzeng and Jeng [14] reported that the cooling performance of heat sink with uncompressed porous media was better than that of compressed media. The effects of bead particle size on the efficiency of heat exchange between the fluid and the solid phases of the heat sink were performed experimentally by Tzeng et al. [15]. The fully developed forced convection in a rectangular microchannel filled with or without a porous medium was examined by Hooman [16]. Convective heat transfer in a channel partially filled with a porous medium was examined by Aguilar-Madera et al. [17]. The above literatures reviewed show that the fluid flow and heat transfer in porous ducts can be improved by a proper porous configuration designs. However, there is only a limited amount of work to investigate the effects of different configuration designs on the thermal performance of the channels, especially for trapezoidal and sandwich configuration designs.

This work is to propose a 3-D computational model of porous-MCHSs with various different porous configuration designs. The effects of the configuration designs on the fluid flow and thermal performance are investigated in details.

\section{ANALYSIS}

Three-dimensional computational domains of the porousMCHSs with six types of porous distribution designs are established in the present study. The porous configuration designs in the MCHSs include the rectangular, outlet enlargement, trapezoidal, thin rectangular, block, and sandwich distribution designs (Fig. 2). The solid part of the 
heat sink and porous medium is made of copper with $k_{\mathrm{s}}=$ $401 \mathrm{~W} \mathrm{~m}^{-1} \mathrm{~K}^{-1}$. Pure water is used as the coolant. Each computational domain consists of a channel, two half-ribs and various porous medium distributions within the channel. Each channel has a height $H_{c}$ and width $W_{c}$, and each vertical rib has a width of $W_{r} / 2$. The thicknesses of the heated wall and cover wall are $\delta_{1}$ and $\delta_{2}$, respectively. The length of the channel in the streamwise direction is $L_{x}$. The coolant flows in the $x$ direction as the pumping power is applied to the coolant. Heat applied on the bottom of the channel is removed via conduction by the porous media and solid walls and then dissipated by convective fluid flowing through the coolant within the porous spaces and the channels. To simplify the analysis, it is assumed that 1) the sintered porous medium is rigid, homogenous, isotropic, and fully saturated with fluid; 2) fluid flow is incompressible, laminar, and steady; 3) local thermal equilibrium between the solid and the liquid phases inside the porous medium; 4) the thermophysical properties of the working materials are constant; 5) the gravity effect and radiation heat transfer are neglected in channels.

The characteristics of the flow and heat transfer for the liquid in the porous media are based on the volumeaveraged technique and Brinkman-Darcy-Forchheimer model of porous media. With the above assumptions, the governing equations of the porous media can be described as follows [2], [9]:

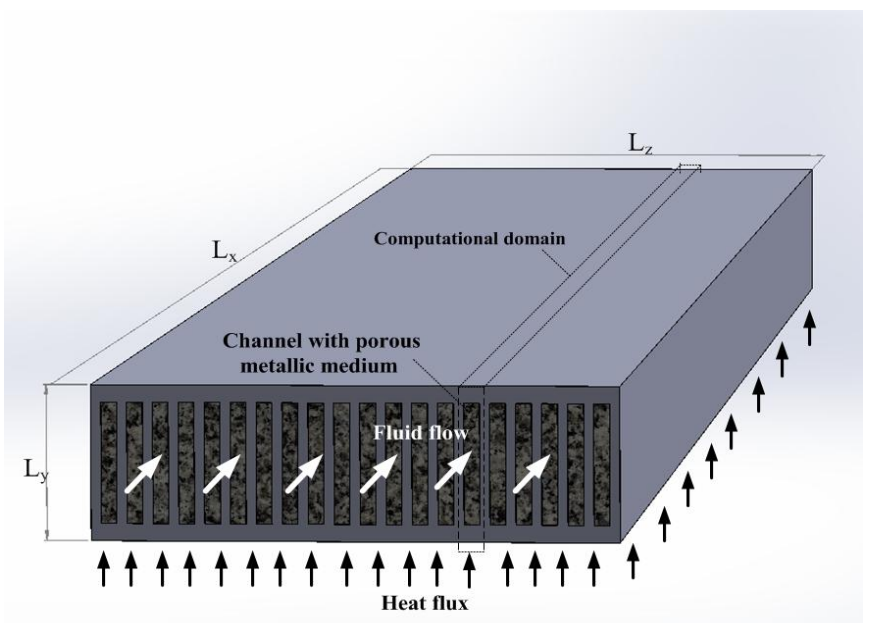

Fig. 1. Schematic diagram of porous-microchannel heat sink (MCHS).

\section{A. Continuity Equation}

$$
\nabla \cdot\left(\varepsilon \rho_{f} \vec{V}\right)=0
$$

where $\rho_{f}$ is the density of the fluid and $\vec{V}$ is the velocity vector of the fluid.

\section{B. Momentum Equations}

$$
\frac{\rho_{f}}{\varepsilon^{2}}(\vec{V} \cdot \nabla) \vec{V}=-\nabla p-\left(\frac{\mu_{f}}{K_{p}}+\frac{\rho_{f} C}{\sqrt{K_{p}}}|\vec{V}|\right) \vec{V}+\frac{\mu_{f}}{\varepsilon} \nabla^{2} \vec{V}
$$

where $\varepsilon$ is porosity of the sintered porous medium; $K_{p}$ is the permeability; $C$ is Forchheimer's constant, and $\mu_{f}$ is the viscosity of the fluid.

\section{Energy Equation}

$$
(\rho c)_{e}(\vec{V} \cdot \nabla T)=k_{e} \nabla^{2} T
$$

where $T$ is temperature, $k_{e}$ and $(\rho c)_{e}$ are the effective thermal conductivity and the heat capacity of the porous media, respectively, are $k_{e}=\varepsilon k_{f}+(1-\varepsilon) k_{s}$ and $(\rho c)_{e}=\varepsilon \rho_{f} c_{f}+(1-\varepsilon) \rho$ ${ }_{s} c_{s}$. The subscript indices $\mathrm{f}$ and $\mathrm{s}$ stand for liquid and solid, respectively.

The governing equations of the solid wall can be written as follows.

$$
0=k_{s} \nabla^{2} T_{s}
$$

where $k_{s}$ is the thermal conductivity of the channel wall.
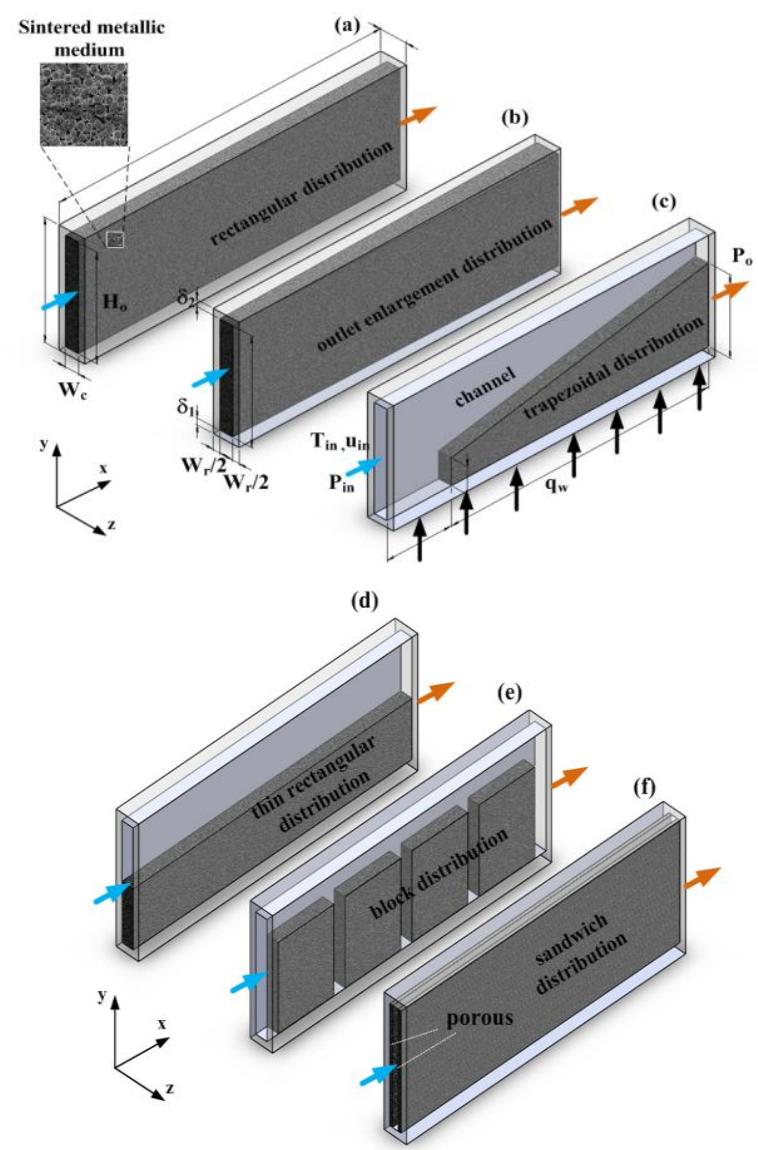

Fig. 2. Schematics of computational domain for (a) rectangular; (b) outlet enlargement; (c) trapezoidal; (d) thin rectangular; (e) block; (f) sandwich distribution design.

Boundary conditions for the computational domain are as follows: the constant velocity is specified, ranging from 0.2 to $6 \mathrm{~m} / \mathrm{s}(\operatorname{Re}=45 \sim 1350)$, at the inlet of the channel. The pressures at the outlets are constant and the flows are fully developed at the outlets of the channels. The uniform inlet temperature of coolant $T_{\text {in }}(293 \mathrm{~K})$ is assumed. At the solid walls, no slip conditions for the velocity are hold. The upper surface of the computational domain is thermally insulated The left and right sides of the domain are assumed to be symmetric. The bottom of the domain is heated with a uniform heat flux $q_{w}$. More details were given elsewhere [20]. 
The pumping power of a channel can be written as

$$
\Omega=\dot{Q} \Delta P=u_{i n} H_{c} W_{c} \Delta P
$$

where $\dot{Q}$ is volume flow rate of the cross-section of the channel.

The dimensionless wall temperature is expressed as

$$
\theta_{w}=\frac{T_{w}-T_{i n}}{q_{w} D_{h} / k_{f}}
$$

The Nusselt number can be written as

$$
N u_{m}=\frac{h_{m} D_{h}}{k_{f}}
$$

with

$$
h_{m}=\frac{q_{w}}{\left(\bar{T}_{w}-T_{i n}\right) A}
$$

where the $T_{\text {in }}$ and $\bar{T}_{w}$ are inlet fluid temperature and the average wall temperature along the centreline of bottom wall, respectively.

\section{NUMERICAL METHOD}

The finite volume method was employed to solve the above governing equations. Details of the numerical solution procedure have been given in the previous studies [9], [20]. Additionally, the coupled set of equations was solved iteratively with convergence criterion being set to be 10-6.

To check the grid independence, three non-uniformly distributed grid configurations are considered. Making the tradeoff between accuracy and execution time, the grid system $50 \times 101 \times 45$ is finally chosen for the simulation model. It is also found that the present predictions agree well with those of Tuckerman and Pease [21] and Ho et al. [22]. Through these tests, the numerical scheme was found to be suitable for the present problem.

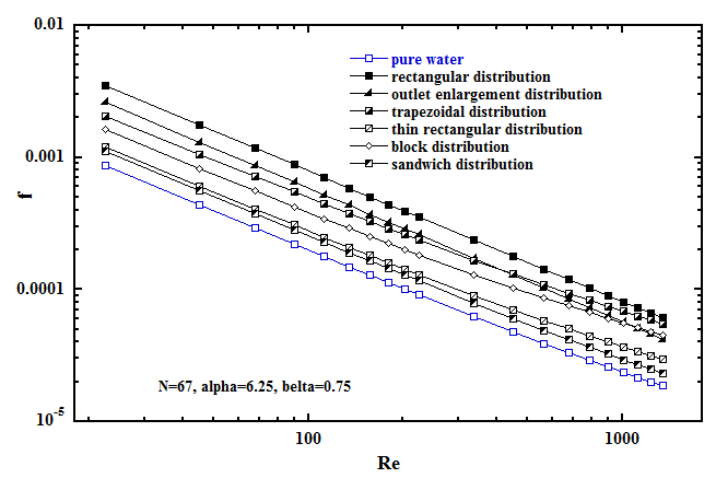

Fig. 3. Comparison of the friction factor with Reynolds number for an MCHS under various porous distribution designs.

\section{RESUlTS AND DisCUSSION}

Fig. 3 shows the variations of the friction factor with Reynolds numbers under various porous distribution designs. Larger friction factors in the channel mean that more powers are needed to pump the coolant. It is clear from Fig. 3 that the friction factor distributions for the MCHSs with different porous configuration designs display a monotonic drop with the Reynolds number, which is larger than that of the nonporous MCHS. It also indicates that the channel with sandwich distribution has the smallest increment in friction factor due to little obstacle in the channel while the channel with a rectangular porous distribution has the biggest one. The results suggest that adding porous medium to the channel will lead to the larger pressure drop, which was accomplished by a drastic increase in pumping power. The lowest pressure drop is noticed for a porous MCHS with sandwich distribution design among the six porous configuration designs.

The effects of porous configuration designs of MCHSs on the average Nusselt number, $N u_{m}$, at various Reynolds numbers are indicated in Fig. 4. Compared with the results without porous medium, the predicted results with different porous configuration designs clearly reveal significant enhancement in Nusselt numbers. The Nusselt number increases with the increasing of the Reynolds number. This enhancement is more obvious compared with the results without porous medium under high Reynolds number. Moreover, under lower Reynolds number, the trapezoidal distribution design has the largest Nusselt number (averagely increased by $85.96 \%$ at $\mathrm{Re}=450$ ) and followed by sandwich distribution design while the thin rectangular has the lowest one. On the other hand, the rectangular distribution has the largest enhancement (averagely increased by $148.05 \%$ at $\mathrm{Re}=1350$ ) in Nusselt number and followed by trapezoidal one under high Reynolds number. Therefore, the Nusselt number can be effectively enhanced by various porous distribution designs. The results confirm that the thermal performance can be enhanced by designing a trapezoidal or sandwich distribution under lower velocity of coolant or selecting a rectangular distribution design under higher one.

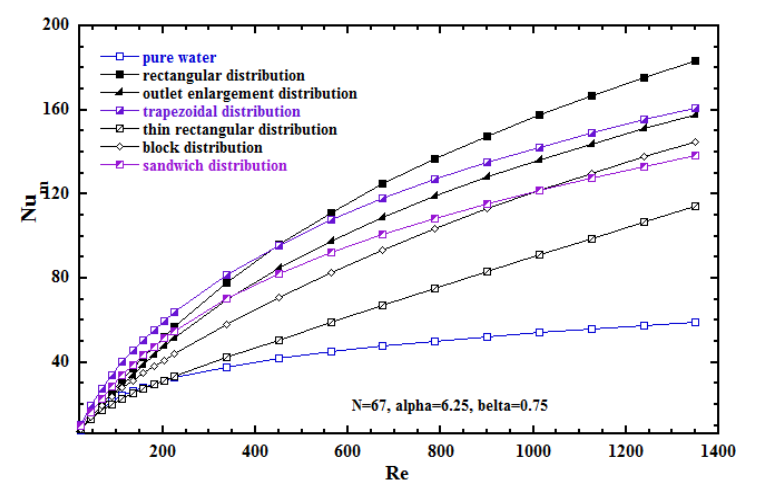

Fig. 4. Variations of average Nusselt number with Reynolds number for a microchannel heat sink under various porous distribution designs.

Such enhancement in the averaged Nusselt number can be further quantified by the corresponding average heat transfer effectiveness $\varepsilon_{h}$, which can be defined as [22]

$$
\varepsilon_{h}=\frac{h_{m, p}}{h_{m, b f}}
$$

where $h_{m, p}$ and $h_{m, b f}$ are the average heat transfer coefficients for the channel with various porous distribution 
designs and the channel without porous medium, respectively. The heat transfer effectiveness is enhanced as the value of $\varepsilon_{h}$ is larger than unity and the larger the value is the larger the heat transfer effectiveness will be. For the conditions considered, the results of $\varepsilon_{h}$ as illustrated in Fig. 5 clearly indicate that adding porous medium in the channel are capable of improving effectiveness in suppression of the bottom wall temperature of the microchannel heat sink for most of designs. In addition, the heat transfer effectiveness increases with the increasing of Reynolds number for all porous configuration designs. Moreover, under lower Reynolds number, the trapezoidal distribution design has the largest heat transfer effectiveness, followed by the sandwich distribution design. On the other hand, the rectangular distribution has the largest enhancement in heat transfer effectiveness under high Reynolds number. In particular, the heat transfer effectiveness is reduced compared with that without porous medium as $\mathrm{Re}<200$ for the thin rectangular distribution design. The main cause of the reduction of the effectiveness may be due to the fact that the pumping power applied could not overcome the larger pressure drop induced by using the thin rectangular distribution design. These results imply that the higher heat transfer effectiveness can be obtained by designing the trapezoidal distribution design at a lower velocity of coolant or selecting the rectangular distribution design at the higher velocity of coolant.

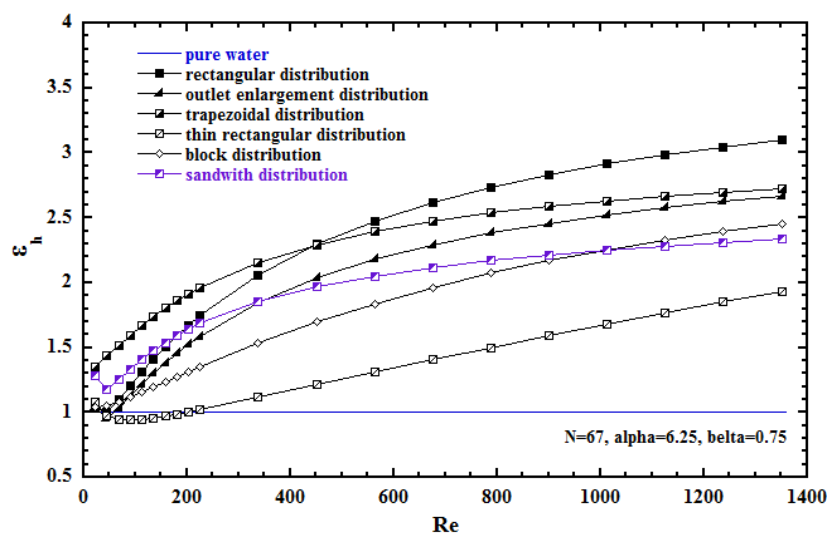

Fig. 5. Variations in average heat transfer effectiveness with Reynolds number for a porous MCHS under various porous distribution designs.

The cooling performance of the heat sink with various porous configuration designs is evaluated by investigating the results of the overall thermal resistance, $R_{T}$, versus the pumping power of a channel as indicated in Fig. 6 . The overall thermal resistance is defined as

$$
R_{T}=\frac{T_{w, \max }-T_{i n}}{q_{w} A}
$$

where $T_{w, \max }$ is the maximum bottom wall temperature of the heat sink and $A$ is the base area of the heat sink. The pumping power was determined from the calculated volume flow rate and pressure drop as Eq. (5). It is observed in Fig. 6 that increasing the pumping power of a channel would drastically reduce the overall thermal resistance for various porous distribution designs. Moreover, the trapezoidal and sandwich distribution designs have the largest decrement of thermal resistance and the more the pumping power is the larger decrement of the thermal resistance will be, compared with the results without porous medium. In particular, the thermal resistances of MCHS with various porous distributions are not necessarily lower than that without porous medium. For instance, the thermal resistances of the rectangular, outlet enlargement, thin rectangular, and block distribution designs are higher than that without porous medium as $\Omega<0.0020 \mathrm{~W}$; For other examples, when $\Omega>$ $0.00045 \mathrm{~W}$ or $\Omega>0.00016 \mathrm{~W}$, the thermal resistance for trapezoidal or sandwich distribution design is far less than that without porous medium. Therefore, from the view point of application, when $\Omega<0.0020 \mathrm{~W}$, the rectangular, outlet enlargement, thin rectangular, and block distribution designs are not available as compared to the results without porous medium. Similarly, when $\Omega<0.00045 \mathrm{~W}$ or $\Omega<$ $0.00016 \mathrm{~W}$, the trapezoidal or sandwich distribution design is not effective. From the above results, it is concluded that the sandwich distribution and the trapezoidal distribution design have the best cooling performance.

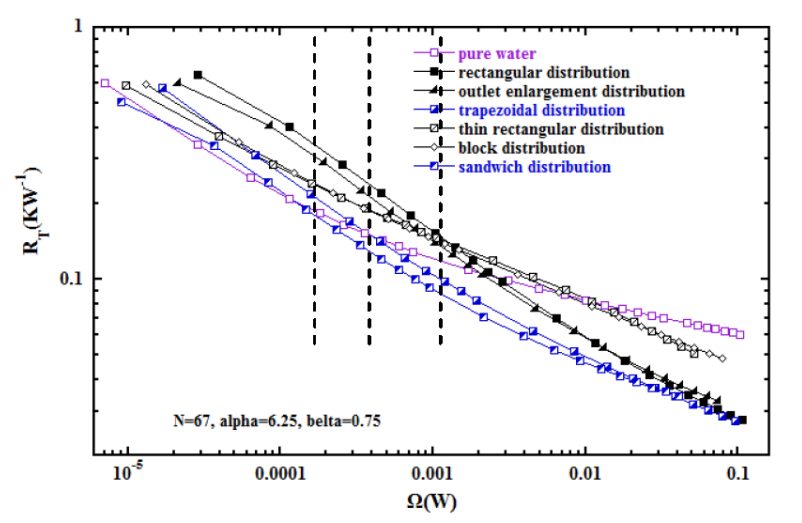

Fig. 6. Comparison of the overall thermal resistance with pumping power for MCHSs under various porous distribution designs.

It can be further demonstrated by the efficacy of using various porous distribution designs in its effectiveness of minimizing the wall temperature, which is of particular interest in temperature control. The temperature control effectiveness can be defined as [22]

$$
\varepsilon_{T_{w}}=1-\frac{\left(T_{w, \max }-T_{i n}\right)_{p}}{\left(T_{w, \text { max }}-T_{i n}\right)_{b f}}
$$

The suppression of the maximum wall temperature (or temperature control effectiveness) of the heat sink is better as the value of $\varepsilon_{T_{w}}$ is larger than 0 and the larger the value is the larger the temperature suppressed will be. Fig. 7 shows that the trapezoidal distribution design has the highest temperature control effectiveness for all the velocity of coolant and followed by the sandwich distribution design at lower Reynolds number. For instance, a suppression of $60 \%$ temperature control effectiveness can be achieved for $\mathrm{Re}=1300$ for the trapezoidal distribution design. On the other hand, the effectiveness for rectangular distribution design is similar to that of the trapezoidal one at higher Reynolds number. In particular, when $\operatorname{Re}<525$ or $\operatorname{Re}<250$, the effectiveness is less than zero for thin rectangular or block distribution design. For instance, the wall temperature increases $18 \%$ as compared to that without porous medium 
for thin rectangular distribution at $\operatorname{Re}=150$. It implies that the temperature control effectiveness is not available under these operating conditions. From the above, the trapezoidal distribution provides a better cooling effectiveness than the other porous designs.

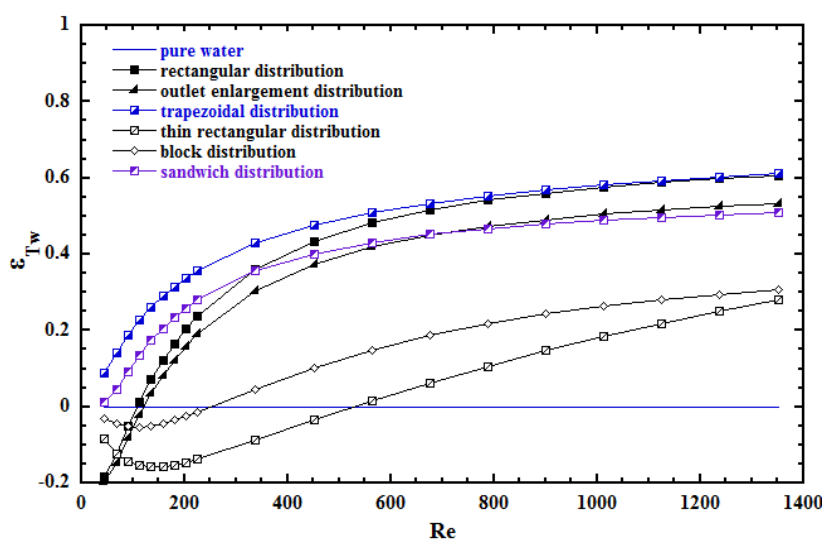

Fig. 7. Variations in temperature control effectiveness with Reynolds number for a porous MCHS under various porous distribution designs.

\section{CONCLUSIONS}

The conclusions drawn from the predicted results are

1) The lowest pressure drop is noticed for a porous MCHS with sandwich distribution design among six configuration design considered.

2) Most of the thermal performances can be enhanced by using the porous configuration designs and they are enhanced with an increase in the Reynolds number, except for lower Reynolds number.

3) The sandwich and the trapezoidal distribution designs have the best heat transfer efficacy and temperature control effectiveness. In particular, the thermal performance for rectangular, outlet enlargement, thin rectangular, or block distribution designs is not necessarily better than that without porous medium under lower pumping power. It may be due to the fact that the pumping power could not overcome the larger pressure drop induced by these porous distribution designs under lower Reynolds number.

4) By considering the thermal performance along with the pressure drop, the sandwich distribution design is the best design of the porous-MCHSs among the six porous configuration designs considered.

\section{ACKNOWLEDGMENT}

The financial support by the National Science Council, R. O. C., through the contract MOST 103-2221-E145-004 is highly appreciated.

\section{REFERENCES}

[1] R. Singh, A. Akbarzadeh, and M. Mochizuki, "Sintered porous heat sink for cooling of high-powered microprocessors for server applications," International Journal of Heat and Mass Transfer, vol. 52, pp. 2289-2299, 2009

[2] T. C. Hung, Y. X. Huang, and W. M. Yan, "Thermal performance analysis of porous-microchannel heat sinks with different configuration designs," International Journal of Heat and Mass Transfer, vol. 66, pp. 235-243, 2013.

[3] V. V. Calmidi and R. L. Mahajan, "Forced convection in high porosity metal foams," ASME J. Heat Transfer, vol. 122, pp. 557-565, 2000.
[4] A. H. Sheikh, D. A. Nield, and K. Hooman, "Heat transfer in the thermal entrance region for flow through rectangular porous passages," International Journal of Heat and Mass Transfer, vol. 49, pp. 3004-3015, 2006.

[5] A. Zehforoosh and S. Hossainpour, "Numerical investigation of pressure drop reduction without surrendering heat transfer enhancement in partially porous channel," International Journal of Thermal Sciences, vol. 49, pp. 1649-1662, 2010.

[6] T. M. Jeng, S. C. Tzeng, and F. Z. Tang, "Fluid flow and heat transfer characteristics of the porous metallic heat sink with a conductive cylinder partially filled in a rectangular channel," International Journal of Heat and Mass Transfer, vol. 53, pp. 4216-4227, 2010.

[7] A. Tamayol, A. Khosla, B. L. Gray, and M. Bahrami, "Creeping flow through ordered arrays of micro-cylinders embedded in a rectangular minichannel," International Journal of Heat and Mass Transfer, vol. 55, pp. 3900-3908, 2012.

[8] F. Alfieri, M. K, Tiwari, I. Zinovik, T. Brunschwiler, B. Michel, and D. Poulikakos, "On the significance of developing boundary layers in integrated water cooled 3D chip stacks," International Journal of Heat and Mass Transfer, vol. 55, pp. 5222-5232, 2012.

[9] Z. M. Wan, J. Liu, K. L. Su, and X. H. Hu, "Flow and heat transfer in porous micro heat sink for thermal management of high power LEDs," Microelectronics Journal, vol. 42, pp. 632-637, 2011.

[10] Y. T. Yang and C. Z. Hwang, "Calculation of turbulent flow and heat transfer in a porous-baffled channel," International Journal of Heat and Mass Transfer, vol. 46, no. 5, pp. 771-780, 2003.

[11] P. X. Jiang, L. Meng, T. J. Lu, L. Yu, and Z. P. Ren, "Experimental Research on convection heat transfer in sintered porous plate channels," International Journal of Heat and Mass Transfer, 2004, vol. 47, pp. 10-11.

[12] P. X. Jiang, L. Meng, T. J. Lu, L. Yu, and Z. P. Ren, "Boundary conditions and wall effect for forced convection heat transfer in sintered porous plate channels," International Journal of Heat and Mass Transfer, 2004, vol. 47, pp. 2073-2083.

[13] G. Hetsroni, M. Gurevich, and R. Rozenblit, "Sintered porous medium heat sink for cooling of high-power mini-devices," International Journal of Heat and Fluid Flow, 2005, vol. 27, no. 2, pp. 259-266.

[14] S. C. Tzeng and T. Jeng, "Interstitial heat transfer coefficient and dispersion conductivity in compressed metal foam heat sinks," ASME J. Electron. Pack., 2007, vo. 129, pp. 113-114.

[15] S. C. Tzeng, W. Y. Jyw, C. W. Lin, and Y. C. Wang, "Mixed convection heat-transfers in a porous channel with sintered copper beads," Applied Energy, vol. 81, no. 1, pp. 19-31, 2005.

[16] K. Hooman, "Heat and fluid flow in a rectangular microchannel filled with a porous medium," International Journal of Heat and Mass Transfer, 2008; vol. 51, pp. 5804-5810, 2008.

[17] C. G. A. Madera, F. J. V. Parada, B. Goyeau, and J. A. O. Tapia, "Convective heat transfer in a channel partially filled with a porous medium," International Journal of Thermal Sciences, vol. 50, pp. 1355-1368, 2011.

[18] I. A. Badruddin, A. A. A. Rashed, N. J. S. Ahmed, and S. Kamangar, "Investigation of heat transfer in square porous-annulus," International Journal of Heat and Mass Transfer, vol. 55, pp. 2184 $2192,2012$.

[19] T. C. Hung, W. M. Yan, and W. P. Li, "Analysis of heat transfer characteristics of double-layered microchannel heat sink," International Journal of Heat and Mass Transfer, vol. 55, pp. 30903099, 2012

[20] S. V. Patankar, Numerical Heat Transfer and Fluid Flow, New York: Hemisphere, 1980

[21] D. B. Tuckerman, "High-performance heat sinking for VLSI," IEEE Electron Device Letter EDL-2, pp. 126-129, 1981.

[22] C. J. Ho and W. C. Chen, "An experimental study on thermal performance of $\mathrm{Al}_{2} \mathrm{O}_{3} /$ water nanofluid in a minichannel heat sink," Applied Thermal Engineering , vol. 50, pp. 516-522, 2013.

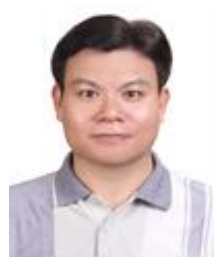

Tu-Chieh Hung is a professor in the Department of Mechanical Engineering at Chinese Military Academy. His current research interests include heat transfer in heat sink nano-fluid, porosity medium, hydrodynamic polishing process, and nanotechnology. He received the BSSB and MSME degrees from Chung Cheng Institute of Technology, Taiwan, from 1986 to 1988, respectively, and the Ph.D. degrees from National Sun Yat-Sen University in Taiwan in 2001. 


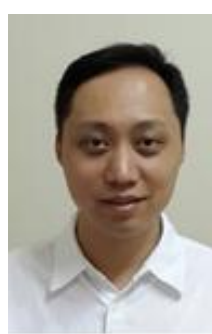

Yu-Xian Huang received his Ph.D. degree in the Department of Mechanical Engineering from National Cheng-Kung University, Tainan, Republic of China, in 2010. He focused on numerical and experimental with fuel cell and reformer channel design in his Ph.D. thesis. Since then, he has worked on load cell structure design as a post-doctoral in Chung-Cheng University within 3 years and thermal analysis for servo motor as a thermal engineering in Delta Electronics, Inc within 2 years, respectively.

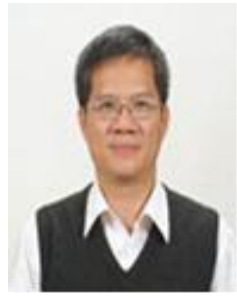

Wei-Mon Yan is a professor in the Department of Energy and Refrigerating Air-Conditioning Engineering at National Taipei University of Technology. He received his M.S. degree in 1985 and the Ph.D degree in 1989 from the Department of Mechanical engineering from National Chiao Tung University. He was selected to be the president of Huafan University. At 2013, he was then invited to join the National Taipei University of Technology. His research interests are focused on transport phenomena of PEM fuel cells and the characteristics of fluid flow and heat transfer in heat sink with nanofluids and MEPCM particles. He has published more than 160 journal papers with an h-index of 30 . 